\title{
Brain-Potential Evidence for the Time Course of Access to Biographical Facts and Names of Familiar Persons
}

\author{
Rasha Abdel Rahman and Werner Sommer \\ Humboldt-University at Berlin
}

\author{
Stefan R. Schweinberger \\ University of Glasgow
}

\begin{abstract}
On seeing familiar persons, biographical (semantic) information is typically retrieved faster and more accurately than name information. Serial stage models explain this pattern by suggesting that access to the name follows the retrieval of semantic information. In contrast, interactive activation and competition (IAC) models hold that both processes start together but name retrieval is slower because of structural peculiarities. With a 2-choice go/no-go procedure based on a semantic and a name-related classification, the authors tested differential predictions of the 2 alternative models for reaction times (RTs) and lateralized readiness potentials (LRP). Both LRP (Experiment 1) and RT (Experiment 2) results are in line with IAC models of face identification and naming.
\end{abstract}

On seeing the face of a familiar person, one may recall many kinds of information about this individual, such as occupation, place of living, or even episodes of life, yet one may be unable to recall the person's name. The relative difficulty of face naming has been frequently demonstrated in reaction-time (RT) experiments (Johnston \& Bruce, 1990; Young, McWeeny, Ellis, \& Hay, 1986), diary studies (e.g., Schweich et al., 1992; Young, Hay, \& Ellis, 1985), learning experiments (McWeeny, Young, Hay, \& Ellis, 1987), and clinical observations (e.g., Flude, Ellis, \& Kay, 1990; Hodges \& Greene, 1998; Semenza \& Zettin, 1989). Typically, access to memory-based knowledge is slower, more difficult, and error prone for names than for other types of information, which we collectively call semantic or biographical knowledge.

Traditionally, the relative disadvantage for name retrieval has been explained by assuming a separate name store that can only be retrieved after some biographical information has been accessed. This view is made explicit in the functional model of face recognition by Bruce and Young (1986), which incorporates name retrieval as the final module within a set of serially arranged discrete processing stages. The perception of a familiar face is assumed to activate structural, view-independent representations of known faces stored in memory (face recognition units; FRU). The FRU in turn is linked to a person identity node (PIN), making available semantic information about the person, and finally, the name node is accessed via the PIN. Note that there is no direct link from the FRU to the representation of a person's name.

Rasha Abdel Rahman and Werner Sommer, Institut für Psychologie, Humboldt-University at Berlin, Berlin, Germany; Stefan R. Schweinberger, Department of Psychology, University of Glasgow, Glasgow, Scotland.

This research was supported by a German Research Council doctoral fellowship (Graduate School 423) to Rasha Abdel Rahman. We appreciate helpful discussions with Mike Burton.

Correspondence concerning this article should be addressed to Rasha Abdel Rahman, who is now at the Max Planck Institute for Psycholinguistics, P.O. Box 310, 6500 AH Nijmegen, The Netherlands. E-mail: rasha.abdel-rahman@mpi.nl
An alternative suggestion has been made in the context of modeling approaches for person recognition. In the interactive activation and competition (IAC) models proposed by Burton and Bruce (1992) and Brédart, Valentine, Calder, and Gassi (1995), each FRU is connected to a specific PIN. In contrast to the Bruce and Young (1986) model, PINs merely serve as modality-free interfaces between FRUs and both the semantic information units (SIUs) as well as representations of proper names. Name representations are conceived either to be included in the SIU pool (Burton and Bruce, 1992) or to be stored separately (Brédart et al., 1995), depending on the variant of the IAC model. The crucial point is that in all IAC models the activation flow from the PINs to the representations of semantic knowledge and names proceeds in parallel. In these models the relative disadvantage for accessing names is caused by the scarcity of excitatory connections from the PINs. Thus, a name representation is usually connected to one PIN only. In contrast, many kinds of semantic information, such as a given nationality or occupation, hold true for several persons. Because the excitatory activation flow between PINs and SIUs is bidirectional, any perceived American actor will activate the SIUs "American" and "actor" and-via excitatory backpropagationsall PINs of other Americans and actors, which in turn further increases SIU-activation. Such excitatory loops do not exist for names because they are usually uniquely connected to one PIN only.

Unfortunately, empirical evidence that distinguishes between serial stage models and IAC models is scant. In fact, most demonstrations of a relative disadvantage of name retrieval can be accommodated by both types of models. Some empirical evidence in favor of independent access to names and semantic information has been provided by a recent priming study by Schweinberger, Burton, and Kelly (2001). Schweinberger et al. primed both semantic and name-phonology decisions about famous faces by either partial semantic or partial name information. Although both primes proved to be effective within their category (i.e., partial semantic information primed semantic decisions, and partial name information primed name-phonology decisions), neither prime re- 
liably affected decisions concerning the other category. Most important, semantic primes did not affect name-phonology decisions. This indicates independent access rather than semantically mediated access to names. The aim of our study was to assess differential predictions of serial stage and IAC models of face identification and naming by recording an event-related brain potential: the lateralized readiness potential (LRP).

\section{The LRP}

The LRP is derived from the readiness potential (RP), a slow negative-going scalp-recorded brain potential that appears several hundred milliseconds prior to voluntary hand movements (Kornhuber \& Deecke, 1965). In choice-response tasks with the two hands, the amplitude of the RP is greater at scalp sites contralateral to the activated hand (e.g., Kutas \& Donchin, 1974; Vaughan, Costa, \& Ritter, 1968). Kutas and Donchin (1980) showed that information about the responding hand is decisive for the beginning of the contralateral dominance of the RP. These observations led to the conclusion that the lateralized aspect of the RP is an index for hand-specific response preparation. The response-side related asymmetry of the RP (i.e., LRP) can be isolated (Gratton, Coles, Sirevaag, Eriksen, \& Donchin, 1988; Smid, Mulder, \& Mulder, 1987) by recording event-related brain potentials from electrodes located above the left and right motor cortices $\left(\mathrm{C}^{\prime}{ }^{\prime}\right.$ and C3', respectively). Then, difference waves are computed time point by time point between these recordings as a function of the response hand appropriate in a given trial. In every trial the recording from the ipsilateral hemisphere is subtracted from contralateral recordings. That is, in a trial in which a left-hand movement is required, the difference wave $\mathrm{C}^{\prime}{ }^{\prime}$ minus $\mathrm{C} 3^{\prime}$ is computed, whereas it is $\mathrm{C}^{\prime}{ }^{\prime}$ minus $\mathrm{C}^{\prime}$ ' for right-hand trials. This procedure eliminates all electrical brain (EEG) activity that is distributed symmetrically across the scalp. The single-trial difference waveforms are then averaged separately for left- and right-hand trials, and in a last step the LRP is computed as the mean of the average difference waves. Deviations of the resulting LRP from the zero line toward increased negativity indicate the activation of the correct response hand at the level of the motor cortex. For a summary of the significance of the LRP see Miller and Hackley (1992).

The LRP has several properties that were used in the present study. It can develop on the basis of partial stimulus information and does not depend on the execution of an overt motor response. For example, Osman, Bashore, Coles, Donchin, and Meyer (1992) and Miller and Hackley (1992) showed LRPs in a two-choice go/no-go task on trials in which the response hand could be selected on the basis of an easily available stimulus dimension (shape) but response execution was tied to a more difficult dimension (size). An LRP was present not only in go trials but also in no-go trials when the response was successfully withheld. This implies the possibility to prepare a response on the basis of initial information even when later information countermands response execution in no-go trials.

Recently, LRP recordings in two-choice go/no-go tasks have been used in psycholinguistic studies that investigated the relative timing of semantic and phonological access in naming common objects (Schmitt, Münte, \& Kutas, 2000; van Turennout, Hagoort, \& Brown, 1997). These studies demonstrated LRPs in no-go trials when response hand was specified by semantic information and the go/no-go decision was determined by name phonology. In contrast, there was no no-go LRP in a complementary condition when name phonology specified response hand and semantics determined the go/no-go decision. These results suggest that the semantic information derived from pictures of common objects is available earlier than name phonology, which was interpreted as evidence for serial access to semantics and name phonology.

In the present study we used a second property of the LRP to study access to biographical facts and names of persons. The LRP can be measured synchronized both to the stimulus as well as to the response (Osman \& Moore, 1993) by synchronizing the waveform during averaging at the moment of either the stimulus onset or the response. The interval between the onset of the stimulus and the beginning of the LRP (S-LRP) indicates the duration of processes that occur prior to central response activation. In contrast, the interval between the onset of the LRP and the response in the response-synchronized LRP (LRP-R) reflects the time course of processes within the interval between central response activation and the execution of the response. This property can be used to functionally localize experimental effects within the information-processing system (e.g., Leuthold, Sommer, \& Ulrich, 1996; Osman \& Moore, 1993) and to investigate the time course of access to semantic and name information in person recognition. To our knowledge, the present study is the first to apply LRP recording in the investigation of face processing.

\section{Experimental Design}

In Experiment 1 the LRP and RTs were recorded while participants performed a two-choice go/no-go task based on semantic and phonological attributes of the persons shown on the screen. In each trial a choice between the left or right response hand was based on a semantic attribute of the person shown (e.g., nationality). This allowed us to record the LRP as an index of central motor activation. Whether the response was to be executed or withheld, the go/no-go decision, depended on a phonological attribute of the person's name. This dual-classification task ensured that in every trial both semantic information as well as name phonology was retrieved. In addition, the presence of an LRP in no-go trials provided additional information about the relative timing of semantic and name retrieval. The most crucial feature of the present experiments is a manipulation of the time demands of semantic access. Whereas in discrete serial stage models any effect of semantic difficulty should always propagate to the naming stage, this does not hold true for IAC models. As we outline below, on the basis of the manipulation of the duration of semantic access, differential predictions for both kinds of models can be made for the LRP. In a second experiment we manipulated the difficulty of both semantic as well as name-phonology classification. Serial stage models predict that both factors have additive effects on RTs. In contrast, if semantic and name information can be retrieved independently, as proposed by IAC models, a reduced or abolished effect of semantic difficulty for the harder phonological classification level is predicted.

\section{Pretest}

To establish stimulus sets that meet the central requirement for the experiments - a reliable difference in RTs for two semantic 
classification tasks - a pretest was conducted. In this pretest conventional two-choice responses to portraits of politicians were required. Each choice response was based on one of several possible dimensions with two exclusive categories each.

\section{Method}

Participants. A total of 24 undergraduates (10 men, 14 women), between ages 21 and $34(M=27)$, took part in the pretest. All participants were right-handed (Oldfield score $>80$; Oldfield, 1971) and had normal or corrected-to-normal visual acuity. Participants were randomly assigned to one of the two experimental groups of equal size.

Stimuli. Two sets of 24 color portraits were compiled, each set consisting of three different shots of eight male politicians from frontal views. The portraits were scanned as 8-bit images with a 256-color scale and edited for homogeneity of background color and all features outside of the face. The horizontal and vertical resolution of the frames was $125 \times 125$ pixels, covering $3.5 \times 3.5 \mathrm{~cm}$. A constant viewing distance of $1 \mathrm{~m}$ was provided by a fixed chin rest. The pictures were presented on a computer screen with a mean luminance of $26 \mathrm{~cd} / \mathrm{m}^{2}$.

The portrayed politicians could be classified on the following dimensions: nationality (foreign or domestic), political party (government or opposition party), initial vowel of surname ( $a$ or $e$ ), and length of surname (two syllables or more). Note that the dimensions nationality and political party are semantic dimensions whereas the others pertain to the names of the portrayed persons. For each dimension the alternative categories were equiprobable; thus, four of the politicians were domestic, four were members of the opposition party, and so forth. In Stimulus Set 1, the dimensions nationality, political party, and initial vowel were orthogonal; in Stimulus Set 2, the dimensions nationality, political party, and length of surname were orthogonal.

Procedure. Each experiment involved a total of 1,728 trials, subdivided into eight blocks that were separated by short breaks. A trial started with the presentation of a fixation cross in the middle of the screen. After $500 \mathrm{~ms}$, the fixation cross disappeared, and a portrait was presented for $1.3 \mathrm{~s}$ with an interstimulus interval of $1.8 \mathrm{~s}$. Participants were instructed to make speeded choice reactions to each stimulus according to the categories of one of the semantic or phonological dimensions. Group 1 performed classifications for the dimensions nationality, political party, and initial vowel. In Group 2, the initial-vowel dimension was replaced by length of surname. The dimensions that were relevant for the classification task were altered from trial block to trial block; the order of classification tasks and the stimulus-response assignment were counterbalanced across participants.

\section{Results and Discussion}

The mean RTs and error rates (see Table 1) were submitted to analyses of variance (ANOVAs), with repeated measures on the three-level variable classification dimension and on the two-level variable stimulus category, representing the alternative levels within each dimension. Where appropriate, epsilon corrections for conservative (Huynh-Feldt; Huynh \& Feldt, 1976) $F$ tests were performed. Separate ANOVAs were performed for each group.

In each group there was a significant main effect of classification dimension, $F(2,22)=6.6, p<.01, \varepsilon=.89$, and $F(2$, $22)=18.5, p<.01, \varepsilon=1.0$, for Group 1 and 2, respectively. Separate comparisons among the classification dimensions revealed that the nationality classification was consistently faster than the political party classification, $F(1,11)=15.8, p<.01$, for Group 1 (mean difference $=56 \mathrm{~ms}$ ) and $F(1,11)=17.2, p<.01$, for Group 2 (mean difference $=111 \mathrm{~ms}$ ). The difference in RTs
Table 1

Mean Reaction Times in ms (and Error Rates in Percentages) in the Pretest

\begin{tabular}{cccc}
\hline & \multicolumn{3}{c}{ Classification task } \\
\cline { 2 - 4 } Group & Nationality & Political party & Name phonology \\
\hline 1 & $611.3(5.9)$ & $667.2(9.5)$ & $671.2(8.1)$ \\
2 & $593.5(5.7)$ & $673.1(8.7)$ & $704.3(9.8)$ \\
\hline
\end{tabular}

Note. Name phonology in Groups 1 and 2 referred to the initial vowel and to the length of the name, respectively.

between political party and name-phonology classification failed to reach statistical significance in both groups, $F(1,11)<1$, for Group 1 (mean difference $=4 \mathrm{~ms}$ ) and $F(1,11)=2.9, p=.1$, for Group 2 (mean difference $=31 \mathrm{~ms}$ ). An ANOVA on the mean error rates, performed separately for both groups with the same variables as for the analysis of RTs, revealed no significant main effect of classification task (both $F_{\mathrm{S}}<2.1$ ).

The pretest confirmed that the stimulus material to be used in the following experiments fulfills the basic requirement of allowing semantic classification tasks that differ in their temporal demands. Thus, the nationality classification is less time demanding (easy semantic task) than the political party classification (hard semantic task).

\section{Experiment 1}

In this experiment, a two-choice go/no-go task was used, where response hand was specified by one of the semantic dimensions assessed in the pretest, whereas the execution or nonexecution of the response depended on the initial-vowel classification of the name. This comparatively fast dimension was chosen because it maximizes differential temporal overlap between semantic and name retrieval as a function of semantic difficulty in the case of independent access.

According to serial stage models the duration of semantic access should have a clear effect on both the stimulus-to-LRP onset interval and RTs. In these models the classification of name phonology is conceived to be contingent on the completion of semantic access. Therefore, any manipulations in the time demand for the semantic classification should propagate across all subsequent stages, including name retrieval. ${ }^{1}$ In contrast, no effect of semantic difficulty is expected for the LRP onset-to-response interval because this interval should be independent of the duration of processes prior to central response-hand activation. Because hand information based on the semantic decision should be available prior to the go/no-go-related phonological information, there should be central response activation even if the execution is withheld and the LRP returns to baseline. The no-go LRP, arising in the case of response inhibition, should be independent of the requirements for semantic classification.

${ }^{1}$ Although semantically mediated access to names might not hold for all kinds of semantic information, the attributes used in our task (nationalityand occupation-related information) are often referred to as obligatory pathways to name codes of familiar faces (e.g., Johnston \& Bruce, 1990; Young et al., 1986). 
IAC models are also compatible with semantic difficulty effects on RT and the onsets of the stimulus-synchronized LRPs. But more important - and unlike serial stage models - they also predict an effect on the interval between LRP onset and the response. This is because the duration of semantic retrieval and classificationon which the elicitation of the LRP depends-increases for the more difficult task, whereas the duration of phonological retrieval and classification - on which response execution depends - is invariant. In addition, as the interval between the semantic handrelated decision and the phonological decision decreases with semantic difficulty, the opportunity for the development of a no-go LRP should decrease also. Therefore two predictions can be made according to IAC models. As semantic difficulty increases, the interval between LRP onset and the response should decrease, and the no-go LRP expected for easy semantic classification should diminish or vanish in more difficult semantic classifications. These effects should not be observed according to serial stage models.

\section{Method}

Participants. The experiment involved 16 participants ( 9 men and 7 women), aged between 19 and $38(M=26)$. All participants were righthanded (Oldfield score $>80$ ) and reported normal hearing and normal or corrected-to-normal vision. For their participation, they received either payment or partial fulfillment of a curriculum requirement. Three participants had to be replaced because their response patterns during data acquisition indicated noncompliance with the instructions.

Procedure. The 24 portraits of Stimulus Set 1 were used, where the dimensions nationality, political party, and initial vowel were orthogonal. All portraits were shown prior to the experiment proper and had to be verbally named and classified according to the semantic dimensions. Participants were accepted only if they knew most of the relevant information; if necessary, missing information was provided by the experimenter.

During the experiment, each trial started with a fixation cross in the middle of the screen. After $500 \mathrm{~ms}$ the fixation cross disappeared and a portrait was presented for 1,300 ms with an interstimulus interval of 1,800 ms. Stimuli demanding right- and left-handed responses were presented equiprobably; however, the relative frequency of the go and no-go stimulus assignment within a given trial block was .8 to .2 , respectively. The high proportion of go trials was aimed to encourage response preparation (Low \& Miller, 1999). Because the assignment of the name categories to execution (go) and nonexecution (no-go) of the response alternated between blocks, all stimulus categories appeared equally often with respect to the whole experiment.

Responses were recorded with two force-sensitive keys mounted behind each other in the midsagittal plane of the participant; the keys were operated with the index fingers. A finger flexion was registered as soon as response force exceeded a criterion of $90 \mathrm{cN}$. In $20 \%$ of the trials the offset of the portrait was followed by a tone after which the politician had to be named verbally. In these trials, the interstimulus interval amounted to 2,600 $\mathrm{ms}$. This requirement was introduced to maintain phonological retrieval and counteract the acquisition of a nonphonological stimulus-response assignment. After false alarms on no-go trials and wrong keypresses and omissions on go trials, participants received written feedback, shown for $300 \mathrm{~ms}$ on the monitor. Participants were advised to avoid eye movements or blinks during the trial blocks.

The experimental session involved a total of 1,632 trials, subdivided evenly into eight trial blocks separated by short breaks. During each trial, we required an easy or hard semantic classification of the portrayed politician to determine the response hand and a phonological classification of the portrayed politician's name for the go/no-go decision. Participants responded by pressing the far or near response key to the nationality or political party of the politician shown. The go/no-go decision was coupled to the initial vowel of the politician's surname ( $a$ or $e$ ). In one half of the experiment participants responded to the nationality of the politician shown, and in the other half they responded to the political party. The order of the semantic conditions was counterbalanced across participants. The assignment of the go and no-go decision to the initial vowel was alternated blockwise in counterbalanced order.

Electrophysiological recordings. The EEG was recorded with $\mathrm{Sn}$ electrodes at Scalp Positions $\mathrm{Fz}, \mathrm{Cz}$, and $\mathrm{Pz}$, as defined by the international 10-20 system (Jasper, 1958), and at $\mathrm{C}^{\prime}{ }^{\prime}$ and $\mathrm{C}^{\prime}$, that is, $4 \mathrm{~cm}$ to the left and right of $\mathrm{Cz}$. All electrodes were referenced to the right mastoid. In addition, the horizontal and vertical electrooculogram was recorded bipolarly from the external canthi (hEOG) and from above and below the midpoint of the right eye (vEOG), respectively. To assess whether there was peripheral response activation during no-go trials, we also recorded the electromyogram (EMG) from standard electrode placements for finger flexor muscles at both forearms (Lippold, 1967). The EMG was rectified and integrated with a time constant of $10 \mathrm{~s}$. The continuously recorded electrophysiological signals were digitized with a rate of 200 samples per second and amplified with a low-pass filter of $30 \mathrm{~Hz}(12 \mathrm{~dB} /$ octave attenuation). Electrode impedance was kept below $5 \mathrm{k} \Omega$ for cephalic sites and below $10 \mathrm{k} \Omega$ for EMG electrodes.

Data analysis. Offline the continuous EEG was intersected into epochs of $1.5 \mathrm{~s}$. Stimulus-synchronized epochs began $200 \mathrm{~ms}$ prior to picture onset and contained 1,300 ms poststimulus activity. Response-synchronized epochs contained an interval of $900-\mathrm{ms}$ pre- and $600-\mathrm{ms}$ postresponse activity. For further analysis, only correct responses within a time window of $1,300 \mathrm{~ms}$ after stimulus onset were considered. Trials with ocular artifacts were discarded. A trial was considered to contain an artifact if the activity in any cephalic recording channel exceeded $50 \mu \mathrm{V}$.

Averaging of electrophysiological signals was performed separately for go and no-go trials and for two conditions of semantic classification. The LRP was calculated according to the procedure described in the introduction. To facilitate comparisons and to assess possible influence on the LRP, we performed for hEOG and EMG a computation analogous to that for deriving the LRP. All stimulus-synchronized waveforms were baseline adjusted by subtracting the average voltage during an interval of $200 \mathrm{~ms}$ preceding the stimulus, and response-synchronized waveforms were adjusted by subtracting the average voltage during a baseline interval of 900 to $700 \mathrm{~ms}$ before the response. The onset latencies of stimulus- and responsesynchronized LRPs were measured with an absolute criterion of $0.4 \mu \mathrm{V}$ below baseline. Latency differences between conditions were statistically assessed with a jackknife-based technique suggested by Miller, Patterson, and Ulrich (1998). This method allows for pairwise comparisons of latency differences between conditions for grand average waveforms rather than for individual-subject LRPs, thus reducing the influence of background noise.

To assess the presence of LRPs on no-go trials, we performed analyses at 20 -ms time intervals, starting $300 \mathrm{~ms}$ after stimulus onset. For each time window two-tailed $t$ tests were performed against zero. A no-go LRP was considered to be present if $t$ tests were significant $(p<.05)$ in four or more successive intervals.

\section{Results and Discussion}

Performance. $\quad$ Paired samples $t$ tests (two-tailed) on mean RTs from correct trials yielded a significant difference between the nationality and the political party classification (666 vs. $697 \mathrm{~ms}$ ), $t(15)=-3.1, p<.01$. In addition, mean error rates were higher for the former than for the latter semantic condition (5.4 vs. 8.7\%), $t(15)=-3.9, p<.01$.

$L R P$. The grand-average stimulus- and response-synchronized LRP waveforms for go and no-go trials are shown in Figure 1. Pairwise comparisons between the easy and hard semantic condition revealed that the $\mathrm{S}$-LRP onset latency increased with semantic difficulty (mean difference $=45 \mathrm{~ms}$ ), $t(15)=2.6, p<.05$. From this we can conclude that the difficulty manipulation of semantic 


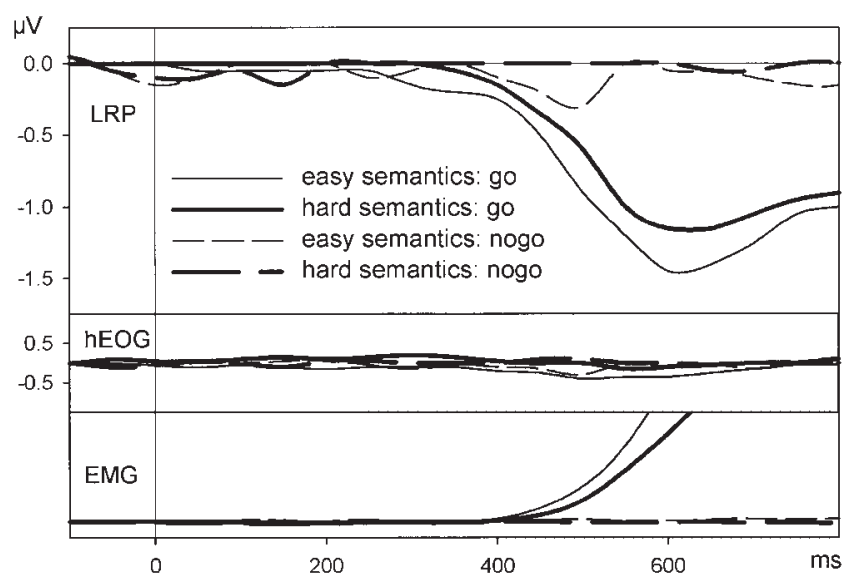

S

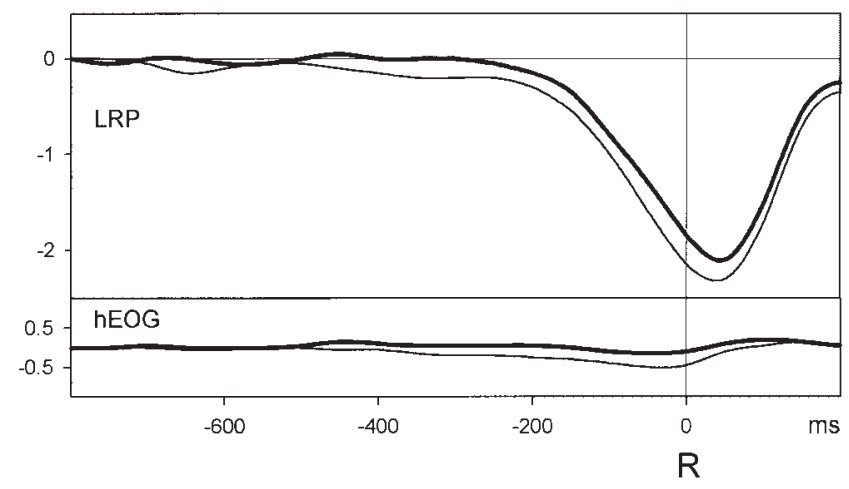

Figure 1. Stimulus- and response-synchronized (top vs. bottom panel) lateralized readiness potential (LRP) and horizontal electrooculogram (hEOG) waveshapes recorded in Experiment 1 as a function of semantic difficulty (easy vs. hard). S and R denote the time points of stimuli and responses, respectively. For the stimulus-synchronized recordings, the electromyogram (EMG) is shown.

access was successful. It is important to note that the interval between LRP onset and the response was about $40 \mathrm{~ms}$ shorter for the hard than for the easy semantic condition, $t(15)=1.8, p<.05$. In the easy semantic condition there was a no-go LRP which deviated significantly from zero between 440 and $520 \mathrm{~ms}$ after stimulus onset, $t \mathrm{~s}(15)>-2.2, p<.05$. In contrast, in the more time-consuming semantic condition (political party classification) a no-go LRP was absent.

As outlined above, serial stage models predict a clear proportional effect of semantic difficulty on RTs. However, the observed effect was small compared with the pretest. Although not itself conclusive, this finding indicates that in the two-choice go/no-go task, the effect of semantic difficulty seen in a mere two-choice task does not simply propagate but may be absorbed to a considerable extent. More important, the observed LRP effects of semantic difficulty are in line with predictions derived from IAC models. According to these models, increasing the interval between the completion of semantic access and name retrieval should be directly reflected in a decreased interval between LRP onset and the response on go trials. In contrast, according to the claim that access to names is coupled to the completion of semantic processing (serial discrete models), the influence of semantic difficulty should not cause any variation in response-synchronized LRP onset latencies. This prediction was disconfirmed by the present data.

Another critical finding is the presence of a no-go LRP for the easy but not for the hard semantic condition. Consistent with the results obtained by van Turennout et al. (1997) a no-go LRP was observed when the response hand was specified by the easy semantic attribute (nationality), but it was absent when the assignment was reversed and name phonology determined response hand (Experiment 1). However, it is a straightforward prediction from serial models that increasing the temporal demands for semantic processing should have no effect on the presence of a no-go LRP; if anything, it might increase the chances for a no-go LRP's appearance when continuous flow of semantic information toward motor stages is assumed. The disappearance of the no-go LRP with increasing semantic difficulty is clearly at variance with predictions of serial stage models. In contrast, this finding agrees with predictions from IAC models. Increasing semantic difficulty diminishes the slack between the termination of semantic and name classification and therefore minimizes the chances for the appearance of a no-go LRP because it arises only when the hand decision (semantics) occurs notably before the go/no-go decision. As the pretest showed, the temporal demands of the hard semantic classification and the vowel classification were in fact very similar, thus obliterating the chances for a no-go LRP to appear. ${ }^{2}$

Why do the onset of the go and no-go LRPs in the easy semantic condition, in which both types of LRP are present, appear to differ, particularly considering that in the van Turennout et al. (1997) study onsets did not differ? This might relate to the relatively low probability of the no-go condition or to differences in the proportion of fast trials in go and no-go conditions that predominate in the determination of onsets (Sommer, Ulrich, \& Leuthold, 1996). In addition, one may be concerned that any no-go LRP in the hard semantic condition might have been obscured by increased variance relative to the easy condition. Such an increase in variance should also hold true for the go condition. An increased variance of single-trial LRPs increases the interval between the LRP onset at its synchronization point because the onset of the averaged LRP is dominated by those single trials that start furthest away in time from the synchronization point (Sommer et al., 1996). However, the interval between go-LRP onset and the response was significantly shorter rather than longer as compared with the easy con-

\footnotetext{
2 These conclusions were further supported by a complementary experiment involving 16 participants, in which the assignment of semantic and name attributes to choice response and execution decision was reversed. For example, participants responded with the right or left index finger to the initial-vowel of the politician shown, whereas the execution-related decision was based on the nationality or political party, respectively. In this experiment, the LRP was coupled to the name-phonology classification. As is predicted by IAC models, but not by serial stage models, the duration of semantic access had no effect on the stimulus-synchronized LRP onset. Furthermore, no-go LRP activity was never observed in any of the semantic conditions. From this we can conclude that the presence of a no-go LRP and of the difficulty effect on both the stimulus-synchronized LRP onset and the LRP-to-response interval in Experiment 1 is not merely an artifact of a task that might induce participants to make the hand and the executionrelated decisions in a fixed order.
} 
dition, ruling out an explanation in terms of increased variance in the more difficult condition.

\section{Experiment 2}

The previous experiment provided evidence from event-related brain potentials that access to semantic knowledge and name phonology does not proceed in series, as proposed in traditional models of face recognition, but is, at least for the concrete semantic attributes used in the present experiment, organized independently. The performance data were not necessarily diagnostic in this respect, although the effect of semantic difficulty in the two-choice go/no-go tasks was conspicuously small as compared with the sizable effect in the two-choice pretest. This discrepancy is compatible with an independent access account that allows an absorption of variations in the time demands of one process during the slack time until the other process is finished. Experiment 2 provided a direct test of the absorption hypothesis by factorially manipulating the difficulty of both semantic access and phonological retrieval. Serial models predict additive effects in which RTs are slowest when both semantic and phonological categorization is hard. In contrast, if semantic access and phonological retrieval are organized in parallel, slack time and thus absorption will depend on the relative duration of these processes. In particular, absorption of any effects of manipulating semantic difficulty should be more pronounced when phonological access is fast as compared with when it is slow. In other words, IAC models would predict an underadditive interaction between semantic and phonological difficulty.

\section{Method}

Participants. Sixteen participants were randomly assigned to two experimental groups of equal size ( 5 men, 3 women, $M=24$ years in Group 1 and 3 men, 5 women, $M=25$ years in Group 2). All participants were right-handed and reported normal hearing and normal or corrected-tonormal vision.

Stimuli. Sets 1 and 2 of the pretest were the stimuli assigned to Group 1 and 2, respectively. The task for Group 1 was the same as in Experiment 1 ; that is, hand decision was dependent on the outcome of a semantic classification (either nationality or political party) and response execution was dependent on the initial vowel of the surname. Group 2 performed the same semantic classifications; however, the name-based classification concerned the number of syllables in the surname (two or less vs. more than two). Performance was statistically assessed with ANOVAs including repeated measurements on semantic difficulty and a group factor of phonological difficulty.

\section{Results and Discussion}

Mean reaction times are shown in Figure 2. In Group 1, which performed the initial-vowel classification, semantic difficulty yielded an RT effect of $71 \mathrm{~ms}, M$ (nationality vs. political party) = 672 versus $743 \mathrm{~ms}$; in contrast, when name classification pertained to the number of syllables, that is, name length, the semanticdifficulty effect was virtually absent, $M$ (nationality vs. political party) $=690$ versus $692 \mathrm{~ms}$. The differential effect of semantic difficulty as a function of the phonological task was confirmed by a two-way interaction of phonological and semantic difficulty in the ANOVA, $F(1,14)=11.3, p<.01, \varepsilon=1.0$. The effect of semantic difficulty in Group 1 alone was strong enough,

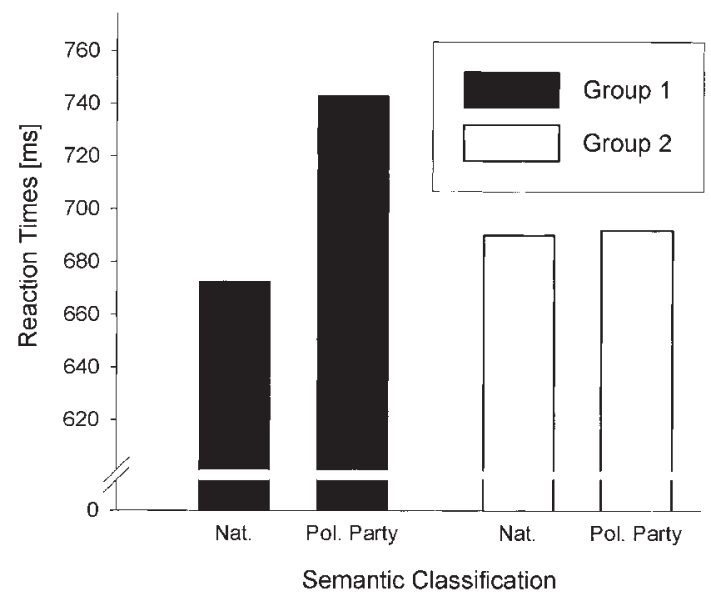

Figure 2. Reaction times from Experiment 2. Group 1 performed an initial vowel classification. Group 2 performed a name length classification. Nat. = nationality; Pol. $=$ political

$t(7)=5.4, p<.01$, to yield a main effect across both groups, $F(1$, $14)=12.8, p<.01$, although the effect was nil in Group 2 alone, $t(7)=0.1$. There was no main effect of the group factor, $F(1$, $14)<1$, nor were there significant differences between the groups at the levels of easy, $t(14)<1$, or hard, $t(14)=1.8, p=.8$, semantic difficulty.

The performance results of Experiment 2 confirm the overall picture presented by Experiment 1 in supporting IAC models of access to semantics and phonology. The effect of semantic difficulty virtually broke down when access to phonology was made more difficult. This was true, although in the particular stimulus set used, the effect of semantic difficulty was very strong (in the order of $100 \mathrm{~ms}$ ) when choice responses were required to the semantic dimension in isolation (cf. pretest). It is quite evident that this finding is completely incompatible with a serial stage model, which would predict (a) effects of semantic difficulty also in the two-choice go/no-go task used here and (b) additivity with any effects of phonological difficulty. Neither prediction was confirmed. In contrast, a decrease of the semantic-difficulty effect at a more difficult, that is time demanding, level of a second variable is well in line with an independent arrangement of the stages manipulated (cf. Stanovich \& Pachella, 1977).

A possible problem in the data of Experiment 2 is that when both decisions are difficult the RTs appear to be somewhat shorter than when only the semantic decision is difficult. It should be noted, however, that this difference was not significant. Even if we took this difference seriously, we could only speculate about its reasons. It might be a group difference in overall response speed or a more specific one for the hard-hard condition; the effect might even relate to processing properties described in the IAC model (e.g., backpropagation of activation). Nevertheless, the overall picture of the present results is in favor of IAC models.

\section{General Discussion}

In Experiment 1, the LRP-to-response interval decreased with increasing semantic difficulty, and a no-go LRP present in the easy semantic task was absent for the hard task. In addition, the effect 
of semantic difficulty in the choice reaction times of the pretest shrank when the task also required phonological classifications. More important, the effect of semantic difficulty vanished completely when the time demands of the phonological decision were further increased (Experiment 2). These findings support parallel and independent accounts of semantic access and name retrieval as suggested in the IAC models of Burton and Bruce (1992) and Brédart et al. (1995). As described in the introduction, these models hold that following the activation of stored structural face descriptions (FRUs), information flows to abstract nodes representing the identity of the person (PINs). From here, the representations for biographical facts and names are activated simultaneously. The present findings unanimously support the suggestion derived from IAC models that as the time demands for semantic fact retrieval increase, the temporal distance to the termination of phonological retrieval diminishes. This conclusion is directly opposed to the invariance of this interval suggested by traditional serial stage models. Whereas previous attempts to empirically distinguish between these types of models have to do with not finding an effect where serial stage models would predict a difference (Schweinberger et al., 2001), the present study reports multiple positive evidence in favor of IAC models.

Might the data pattern reported in Experiment 1 be explained also by continuous information flow from a semantic level to a phonological level, as suggested by cascade models (e.g., McClelland, 1979)? According to cascade models, increasing difficulty of semantic access may slow the rate function of subsequent phonological processing. As a result, both go and no-go LRP waveshapes should rise more slowly in the hard than in the easy semantic condition. In addition, the no-go LRP should be preserved even in the hard semantic condition because as a result of rate limitation at the phonological level, the following motoric processes should also be slowed. Both predictions are at variance with the data.

If, on the other hand, semantic difficulty has no rate-limiting effects on both phonological and motoric levels - an alternative possibility in cascade models - the difficulty manipulation should not affect the LRP at all. In addition, if phonological encoding, but not motoric activation, is rate limited by semantic difficulty, there should be no-go LRPs both in the easy as well as in the hard semantic condition, which is a prediction also not borne out by our data.

In the present design a small set of stimuli was used. Therefore participants might have acquired direct associations between stimuli and responses rather than retrieving semantic and name information in each trial. Our precautions against this risk were the use of several portraits for each person and frequent verbal naming of the portrayed. To the extent that participants had acquired stimulus-response associations despite these precautions, semantic difficulty effects should have been obliterated. The persistence of several such effects argues against an overruling influence of stimulus-response associations in the present data.

Might the present data pattern relate to the possibility that the initial-vowel decision required for names is qualitatively different, for example, by being more fine grained than the semantic decisions? In our opinion, this might at most concern the pretest, where name-related decisions were compared with semantic decisions and which, incidentally, shows very similar patterns to what is commonly observed when naming latencies are measured (cf. Johnston \& Bruce, 1990; Young et al., 1986). The concern is unlikely to question the validity of the experiments proper because here task was kept constant and only the effects of semantic difficulty were assessed.

Defenders of serial stage models might accept the current findings as supporting an IAC model but suggest that such an architecture may not hold for all kinds of information. It might be argued that at least some aspects of semantic knowledge are obligatorily accessed prior to the activation of other kinds of biographical and name-related facts. Although, to our knowledge no such suggestions have been made in the domain of person recognition, it is an option that can be investigated with the present type of design.

In conclusion, by combining a two-choice go/no-go task with the manipulation of the temporal demands of semantic retrieval, we assessed differential predictions derived from serial stage and IAC models for the access to semantic knowledge and name after seeing the face of a familiar person. Both the performance and brain-potential evidence was in favor of IAC models. The present approach appears to be a powerful tool to assess competing models of information processing.

\section{References}

Brédart, S., Valentine, T., Calder, A., \& Gassi, L. (1995). An interactive activation model of face naming. Quarterly Journal of Experimental Psychology, 48(A), 466-486.

Bruce, V., \& Young, A. (1986). Understanding face recognition. British Journal of Psychology, 77, 305-327.

Burton, A. M., \& Bruce, V. (1992). I recognize your face but I can't remember your name: A simple explanation? British Journal of Psychology, 83, 45-60.

Flude, B., Ellis, A., \& Kay, J. (1990). Face processing and name retrieval in an anomic aphasic: Names are stored separately from semantic information about people. Brain and Cognition, 11, 60-72.

Gratton, G., Coles, M. G. H., Sirevaag, E. J., Eriksen, C. W., \& Donchin, E. (1988). Pre- and poststimulus activation of response channels: A psychophysiological analysis. Journal of Experimental Psychology: Human Perception and Performance, 14, 331-344.

Hodges, J. R., \& Greene, J. D. W. (1998). Knowing about people and naming them: Can Alzheimer's disease patients do one without the other? Quarterly Journal of Experimental Psychology, 51(A), 121-134.

Huynh, H., \& Feldt, L. S. (1976). Estimation of the box correction for degrees of freedom from sample data in the randomized block and split-plot designs. Journal of Educational Statistics, 1, 69-82.

Jasper, H. H. (1958). Report of the committee on methods of clinical examination in electroencephalography. Electroencephalography and Clinical Neurophysiology, 10, 370-375.

Johnston, R. A., \& Bruce, V. (1990). Lost properties? Retrieval differences between name codes and semantic codes for familiar people. Psychological Research 52, 62-67.

Kornhuber, H. H., \& Deecke, L. (1965). Hirnpotentialaenderungen bei Willkuerbewegungen und passiven Bewegungen des Menschen: Bereitschaftspotential und reafferente Potentiale [Brain potential changes for voluntary and passive movements in humans: Readiness potential and afferent potentials]. Pfluegers Archiv fuer die Gesamte Physiologie, 284, $1-17$.

Kutas, M., \& Donchin, E. (1974, November 8). Studies of squeezing: Handedness, responding hand, response force, and asymmetry of readiness potential. Science, 186, 545-547.

Kutas, M., \& Donchin, E. (1980). Preparation to respond as manifested by movement-related brain potentials. Brain Research, 202, 95-115.

Leuthold, H., Sommer, W., \& Ulrich, R. (1996). Partial advance informa- 
tion and response preparation: Inferences from the lateralized readiness potential. Journal of Experimental Psychology: General, 125, 307-323.

Lippold, O. C. J. (1967). Electromyography. In P. H. Venables \& I. Martin (Eds.), A manual of psychophysiological methods (pp. 245-298). Amsterdam: North-Holland.

Low, K. A., \& Miller, J. (1999). The usefulness of partial information: Effects of go probability in the choice/nogo task. Psychophysiology, 36, 288-297.

McClelland, J. L. (1979). On the time relations of mental processes: An examination of systems of processes in cascade. Psychological Review, 86, 287-325.

McWeeny, K. H., Young, A. W., Hay, D. C., \& Ellis, A. W. (1987). Putting names to faces. British Journal of Psychology, 78, 143-149.

Miller, J., \& Hackley, S. A. (1992). Electrophysiological evidence for temporal overlap among contingent mental processes. Journal of Experimental Psychology: General, 121, 195-209.

Miller, J., Patterson, T., \& Ulrich, R. (1998). A Jackknife-based method for measuring LRP onset latency differences. Psychophysiology, 35, 99115.

Oldfield, R. C. (1971). The assessment and analysis of handedness: The Edinburgh inventory. Neuropsychologia, 9, 97-113.

Osman, A., Bashore, T. R., Coles, M. G. H., Donchin, E., \& Meyer, D. E. (1992). On the transmission of partial information: Inferences from movement-related brain potentials. Journal of Experimental Psychology: Human Perception and Performance, 18, 217-232.

Osman, A., \& Moore, C. M. (1993). The locus of dual-task interference: Psychological refractory effects on movement-related brain potentials. Journal of Experimental Psychology: Human Perception and Performance, 19, 1292-1312.

Schmitt, B. M., Münte, T. F., \& Kutas, M. (2000). Electrophysiological estimates of the time course of semantic and phonological encoding during implicit picture naming. Psychophysiology, 37, 473-484.

Schweich, M., Van der Linden, M., Brédart, S., Bruyer, R., Nelles, B., \& Schils, J.-P. (1992). Daily-life difficulties in person recognition reported by young and elderly subjects. Applied Cognitive Psychology, 6, 161172 .
Schweinberger, S. R., Burton, A. M., \& Kelly, S. W. (2001). Priming the access to names of famous faces. British Journal of Psychology, 92, 303-317.

Semenza, C., \& Zettin, M. (1989, December 7). Evidence from aphasia for the role of proper names as pure referring expressions. Nature, 342, $678-679$.

Smid, H. G. O. M., Mulder, G., \& Mulder, L. J. M. (1987). The continuous flow model revisited: Perceptual and central motor aspects. In R. Johnson, Jr., J. W. Rohrbaugh, \& R. Parasuraman (Eds.), Current trends in event-related potential research (EEG Suppl. 40, pp. 270-278). Amsterdam: Elsevier.

Sommer, W., Ulrich, R., \& Leuthold, H. (1996). Das Lateralisierte Bereitschaftspotential als psychophysiologischer Zugang bei der Untersuchung kognitiver Prozesse [The lateralized readiness potential as psychophysiological approach to the investigation of cognitive processes]. Psychologische Rundschau, 47, 1-14.

Stanovich, K. E., \& Pachella, P. G. (1977). Encoding, stimulus-response compatibility, and stages of processing. Journal of Experimental Psychology: Human Perception and Performance, 3, 411-421.

van Turennout, M., Hagoort, P., \& Brown, C. M. (1997). Electrophysiological evidence on the time course of semantic and phonological processes in speech production. Journal of Experimental Psychology: Learning, Memory, and Cognition, 23, 787-806.

Vaughan, H. G., Jr., Costa, L. D., \& Ritter, W. (1968). Topography of the human motor potential. Electroencephalography and Clinical Neurophysiology, 25, 1-10.

Young, A. W., Hay, D. C., \& Ellis, A. W. (1985). The faces that launched a thousand slips: Everyday difficulties in recognizing people. British Journal of Psychology, 76, 495-523.

Young, A. W., McWeeny, K. H., Ellis, A. W., \& Hay, D. C. (1986). Naming and categorizing faces and written names. Quarterly Journal of Experimental Psychology, 38(A), 297-318.

Received July 6, 2000

Revision received May 21, 2001

Accepted July 25, 2001 